\title{
A Fault Diagnostic tool based on a First Principle Model Simulator
}

\author{
F. Cannarile ${ }^{1,2}$, M. Compare ${ }^{1,2}$, E. Zio ${ }^{1,2,3}$ \\ ${ }^{1}$ Aramis Srl, Milano, Italy \\ ${ }^{2}$ Energy Department, Politecnico di Milano, Milano, Italy \\ ${ }^{3}$ Chair on Systems Science and the Energetic Challenge, Fondation EDF, Ecole \\ Centrale Paris and Supelec, France
}

\begin{abstract}
We develop a First Principle Model (FPM) simulator of a solenoid micro-valve of the control system of a train braking system. This is used for failure diagnostic when field data of normal and abnormal system behaviors are lacking. A procedure is proposed to adjust the diagnostic model once field data are available.
\end{abstract}

Keywords: Fault Diagnostics; Feature Selection; Wrapper Approach; Solenoid Valve; Train Braking System.

\section{Introduction}

The engineering field that focuses on Detection, Diagnostics and Prognostics is often referred to as Prognostics and Health Management (PHM) (Zio, 2012). PHM allows for a significant reduction in the system unavailability through an efficient and agile maintenance management, capable of providing the right part to the right place at the right time, together with the necessary resources to perform the maintenance task (Pipe, 2008). This has obviously gained the interest of industry and has resulted in new winwin maintenance service contract models.

This new situation has led several companies of different industrial sectors (i.e., transport, aviation, energy, etc.) to look into PHM first of all simply because having a PHM-equipped system may be the mandatory condition to sell it and, then, because there may be new important sources of income, related to the new opportunities arising if the company is able to sell added values by taking over parts of clients' business risks and other (financial) burdens. In this way, a contractor can diversify his "product" range and may be able to achieve a higher profit.

On the other side, the companies interested in PHM have to balance the risks of PHM due to the lack of experience and the capital expenditures required to purchase the 
necessary instrumentation, software and specialized knowledge. These risks and costs are larger at the beginning of the development of PHM, when real data of normal and abnormal system behaviors are lacking or scarce, and in case of new systems, when there is no experience on their operation. In these situations, the capability of PHM is difficult to be estimated, and this may lead potentially interested companies to distrust the investment in developing a PHM solution.

One possible way to overcome the initial skepticism of the companies is to propose an adaptive and robust PHM development framework, which allows updating and adjusting the PHM tool on the basis of the Knowledge, Information and Data (KID) that incrementally become available as the development goes on from the design to its operation in different working and aging conditions. This gives the companies the possibility of tracking the development of the PHM and the improvement of its performances, which are, then, partially reassured about the risks undertaken in the different stages.

In this work, we present a case study concerning a solenoid valve mounted on the braking system of trains, which has been proposed by a company of the transportation industry interested in fault diagnostics. The case study is characterized by the fact that real data patterns of both normal and abnormal solenoid valves behaviors are currently unavailable. Thus, to start the PHM development pathway, we have developed a First Principle Model (FPM) simulator, which gives a sound basis for an initial identification an optimal subset of features for the development of a diagnostic classifier with high classification performance.

This work paves the way to future works, when real signal measurements from the solenoid valve become available: the developed algorithms will be adaptively tuned and their parameters updated to provide more accurate and precise estimates, and, further, field data will be exploited to take into account uncertainty related to unmodelled dynamics. For this, we also sketch the entire development pathway for building a diagnostic tool, from the FPM development up to the tuning of the diagnostic classifier based on real data.

Obviously, the simulator can also be used as a reliability-based design tool, for designing solenoid valves meeting given reliability requirements with a specific confidence, and as a workbench to design tests on the valve.

The paper is organized as follows: in Section 2, the FPM-based fault diagnostic tool is presented in details; Section 3 details the FPM of the solenoid valve; Section 4 illustrates the development of the diagnostic system based on the FPM. Section 5 concludes the work.

\section{Diagnostic tool based on FPM simulator}

In this Section, we present our methodology for the fault diagnostic issue. The main tasks to be addressed by the diagnostic tool are:

- Detect anomalous conditions at an early stage of the initiation of the degradation processes (Fault Detection), for problem awareness. 
- Characterize the detected conditions (Fault Diagnostics), for establishing the correct maintenance action.

- Quantify the uncertainty on the Detection and Diagnostics outcomes.

These tasks are pursued by implementing the procedure detailed in the following Subsections. Notice that in general, fault detection and fault diagnostics are tackled separately, whereas our approach these tasks are properly integrated.

\subsection{Fault detection procedure}

A general procedure to tackle the fault detection issue is made up of the following steps:

S1) Building a dataset (data history matrix) containing the signals corresponding to the normal system operation. To do this while field data are unavailable, either a first principle model (FPM) or a parametric model (PM), (Zio, 2012) is developed and simulated in the expected normal operating conditions.

S2) Feature extraction and selection: The aims of this step are: a) the extraction of features from the simulated Condition Monitoring (CM) signals and b) the selection of an optimal subset of features to be used for fault detection. For more details, see, for example (Baraldi et al., 2016).

S3) Fault detection: Two different strategies can be pursued to address the fault detection issue, which are referred to as "Strategy A" and "Strategy B".

\section{Strategy A}

This strategy is based on the development of an Empirical Classification Model (ECM), which builds a mapping function between signal features coming from step S2) and degradation levels (normal condition or abnormal condition).

S3A) Fault detection: Build an empirical detection classification model (e.g., Support Vector Machine (SVM), Fuzzy K-Nearest Neighbors (FKNN), Artificial Neural networks (ANN), etc., (Hastie et al., 2009)) based on the features selected at step S2. The assumption here is that the values of the features corresponding to normal conditions are different from those corresponding to abnormal conditions.

\section{Strategy B}

This strategy is based on the development of an Empirical Reconstruction Model (ERM) (Baraldi et al., 2011), which reconstructs the values of the features expected in the component normal conditions: features are extracted from signals observed during operation and compared with the reconstruction provided by the ERM; then, abnormal component conditions are detected when the reconstructions are remarkably different from measurements (Baraldi et al., 2011).

S3B) Normal operation data reconstruction: the aim of this step is to build an ERM (e.g., based on non-parametric Auto-Associative Kernel Regression (AAKR), Artificial Neural Network (ANN), Self Organizing Maps (SOMs) Fuzzy Similarity Analysis (FSA)) to reconstruct the values of the features corresponding to normal operation based on the optimal subset selected at step S2, even if the measured ones correspond to faulty conditions. 
S3B) Residual generation; compute the residuals between the measured data and the normal operation data reconstructed at step S3B.

S3B) Fault detection: develop an algorithm, e.g., Sequential Probability Ratio Test (SPRT) (Baraldi et al., 2010), to perform fault detection. The detection is typically alarmed when the distance between the signal feature values corresponding to the normal operating conditions and their actual values achieve a given threshold. Finally, the uncertainty in the distance estimation is also evaluated, which comes from both the lack of knowledge and the stochastic behavior of the signals. This allows controlling the robustness and the confidence on the maintenance decisions made upon the alarm.

S4) Fault history matrix update: as normal operation data is collected on the component/system (in lab experiments or in the field), the data history matrix built at step S1 is updated by appending the new data.

S5) FPM calibration. This activity makes use of as much information as possible from well-designed laboratory or pilot-plant experiments to obtain the most accurate characterization of the fundamental physics of the process involving the continual adjustment of the model and its parameters. The problem of estimating the FPM parameters from real field data can be framed as an inverse problem. The two currently predominant approaches to parameter estimation are those based on Particle Filtering (PF, Kantas et al., 2015), and Moving Horizon Estimation (MHE, Kühl et al, 2011).

S6) Fault detection model updating: this step requires relaunching the feature selection algorithm in the light of the updated fault history matrix (step S4) and FPM parameters.

S7) Uncertainty Analysis: see Subsection 2.3.

\subsection{Fault Diagnostics}

D1) Faulty operation data history matrix generation: while field data are unavailable, inject faults in the FPM to simulate faulty operation data. This allows creating the faulty operation data history matrix generation.

D2) Feature extraction and selection as described in S2.

D3) Fault diagnostics: build a fault classification model in a similar manner, as illustrated in S3B.

D4) Fault data history matrix updating. As faulty operation data is collected (in lab experiments or in the field), update the data history matrix generated in D1, similarly to what is done in S4.

D5) FPM calibration as described in S5.

D6) Fault diagnostic model updating as described in S6.

D7) Uncertainty Analysis: see Subsection 2.3.

Notice that our approach to fault diagnostics is based on the development of an ECS as in "Strategy A" for fault detection. Therefore, this can be easily integrated with diagnostics by letting steps D1-D2-D3-D4 above be preceded by step S1.

\subsection{Uncertainty Analysis}


When field data becomes available, i.e., after steps S4 and D4, this can be used to take into account unmodeled dynamics due to epistemic uncertainty related to the nature of the measurement apparatus, assumptions made by the experimenter, and the FPM used to make inferences based on the observed data. Different representations of epistemic uncertainty are possible: probability bound analysis, imprecise probability, evidence theory and possibility theory. (Aven \& al., 2014). Once uncertainty has been represented using the approaches listed above, it must be propagated to the quantity of interest (e.g., the predicted RUL). Methods for uncertainty propagation are described in detail in (Aven \& al., 2014).

\section{$3 \quad$ FPM simulator for solenoid valves}

The application of our approach to fault diagnostics to a pilot case is proposed in this Section. The equipment considered are solenoid valves mounted on the control system of train backing systems. Steps S1, D1, D2 and D3 of the procedure described above are implemented. Specifically, "Strategy A" for fault detection is applied and integrated with fault diagnostics. Namely, we first develop a FPM model for normal behavior (step S1), which is exploited to simulate the valve behavior and acquire the solenoid current signals. Then, the model of the degradation process due to the accumulation of debris at the valve seat is embedded into the FPM simulator. This mechanism of degradation behaves as a continuous-state stochastic process, and it has been approximated as a discrete-state process. The simulation of the degradation mechanism together with the valve behavior allows acquiring the signals corresponding to the different behaviors of the solenoid valve in the different degradation states. A FKNN classification algorithm, then, is developed for diagnostics (steps D2 and D3), based on the simulated solenoid current signals. The development of the classification algorithm (step D3) has been integrated with a wrapper method for optimal feature selection (step D2), based on the Differential Evolution technique (Baraldi et al., 2016). Figure 1 summarizes the application of steps (S1), (D1), (D2) and (D3) of the proposed methodology to the solenoid valve case study. 


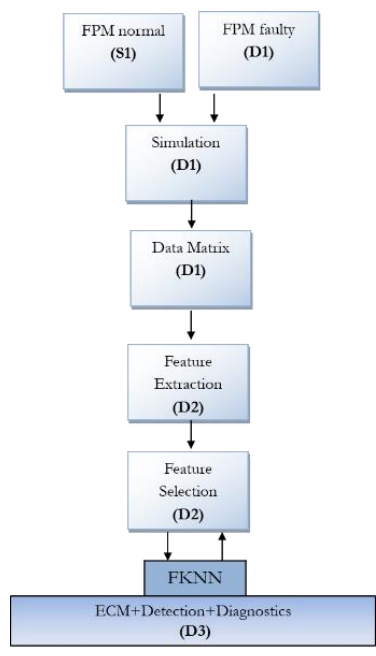

Fig. 1. Scheme for fault detection and diagnostics of the solenoid valve

\subsection{Solenoid valves First Principle Model (Step S1 and D1)}

In this Subsection, the FPM of the solenoid valve under study is presented. The solenoid valve here considered is a fast switching spring return $3 / 2$ valve which is used in the Normally Open (NO) setting (Daigle \& Goeble, 2010).

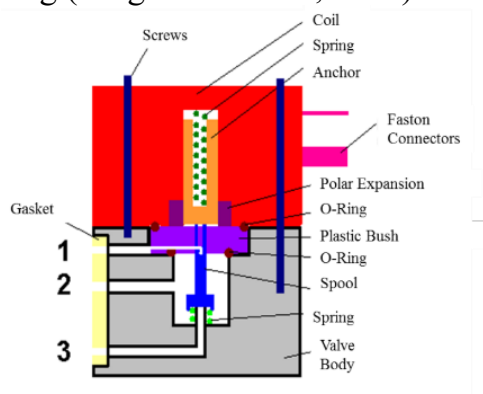

Fig. 2. Solenoid $3 / 2$ valve

The solenoid valve can be framed as made up of three interacting subsystems: magnetic, electric and mechanic subsystems (Taghizadeh et al., 2009). In the following, these are deeply investigated and the governing equations are derived in the form of nonlinear state equations for a NO valve. The objective is to build a valve model that is accurate, relies on a reasonable number of parameters, and can be used as an effective simulation tool.

\subsubsection{Magnetic Subsystem}


The magnetic circuit consists of two parts (Figure 3):

- a fixed core surrounded by $N$ coil turns carrying a current $i$, and

- a moving part, called cylindrical air gap, which moves under the effect of the exerted magnetic force $F_{m}$.

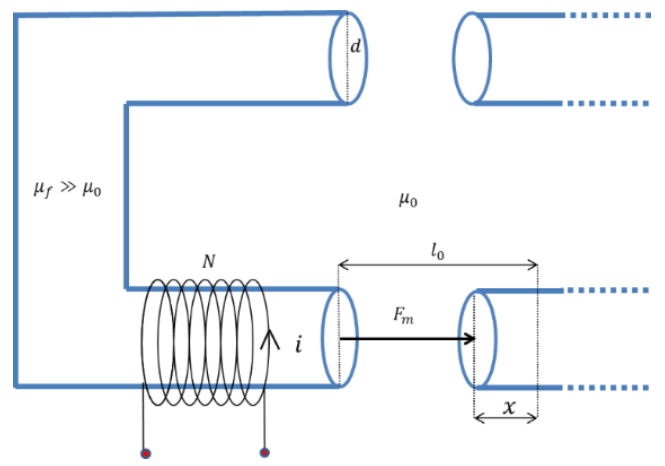

Fig. 3. Model of the magnetic circuit

The quantities appearing in Figure 3 are:

- $\mu_{0}$ : magnetic permeability in the vacuum.

- $\mu_{r}$ : magnetic permeability of the core.

- $d$ : pole diameter of the cylinders.

- $l_{0}$ : air gap total length.

- $x$ : decrease in the length of the air gap due to the magnetic force.

Assuming that the reluctance of the core is negligible $\left(\mu_{f} \gg \mu_{o}\right)$, the magneto-motive force $\mathcal{F}$ can be written as (Rizzoni, 2007):

$$
\mathcal{F}=N i=\mathcal{R}_{\text {gap }} \phi
$$

where $\mathcal{R}_{\text {gap }}$ is the reluctance of the air gap and $\phi$ is the magnetic flux. For a cylindrical air gap, the reluctance is given by (Rizzoni, 2007):

$$
\mathcal{R}_{\text {gap }}=\frac{l_{0}-x}{\mu_{0} A}
$$

where

$$
A=\frac{\pi d^{2}}{4}
$$

The magnetic force in the air gap is given by (Rizzoni, 2007):

$$
F_{m}=\frac{\phi^{2}}{2} \frac{\partial R_{g a p}(x)}{\partial x}
$$

Eq. (1) and Eq. (2) yield: 


$$
\phi=\frac{N i}{R_{g a p}}=\frac{N i \mu_{0} A}{l_{0}-x}
$$

Considering that

$$
\frac{\partial R_{g a p}(x)}{\partial x}=\frac{-1}{\mu_{0} A}
$$

it follows that

$$
F_{m}=\frac{\phi^{2}}{2} \frac{\partial R_{g a p}(x)}{\partial x}=-\frac{N^{2} i^{2} \mu_{0} A}{2\left(l_{0}-x\right)^{2}}
$$

This force acts on the shuttle of the valve, as shown in the mechanical subsystem described in Subsection 3.3.

\subsubsection{Electric Subsystem}

The equivalent electric circuit of the micro-valve is schematized in Figure 4.

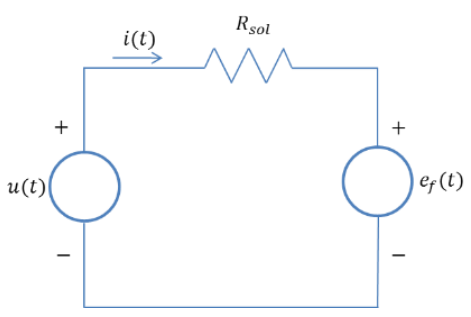

Fig. 4. Model of the electric circuit

By applying Kirchhoff's voltage law, the state equation of this subsystem can be written by

$$
u(t)=R_{\text {sol }} i(t)+e_{f}(t)
$$

in which $u(t)$ is the input voltage, $R_{\text {sol }}$ is the solenoid resistance and $e_{f}(t)$ is the electromotive force induced by the changing magnetic field. According to Faraday's law, $e_{f}(t)$ is given by (Rizzoni, 2007):

$$
e_{f}(t)=\frac{d \lambda}{d t}
$$

where $\lambda=N \phi$ is the flux linkage, which is also equal to $L \cdot i$. The inductance of the magnetic circuit, which determines the value of $e_{f}(t)$, can be derived from the expression of the magnetic flux $\phi$ in Eq. (5):

$$
L(x)=\frac{N^{2}}{R_{g a p}(x)}
$$

Then, Eq. (8) can be written as: 


$$
\frac{d i(t)}{d t}=\frac{1}{L(x)}\left(u(t)-R_{s o l} i(t)-i(t) \frac{\partial L(x)}{\partial x} \frac{d x}{d t}\right), i(0)=i_{0}
$$

\subsubsection{Mechanical Subsystem}

The mechanical subsystem consists of a spring with spring rate $K_{1}$, a mass $m$ (i.e., the spool mass) and a second spring with spring rate $K_{2}$. The mechanical system is shown in Figure 5.

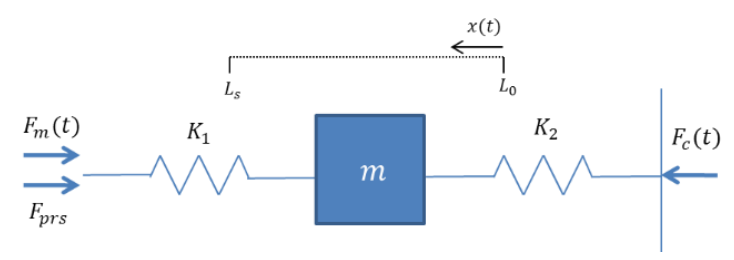

Fig. 5. Model of the mechanical subsystem

The term $F_{c}(t)$ in Figure 5 represents the contact force with the seat (Daigle \& Goeble, 2010), which is described by:

$$
F_{c}(t)=F_{c}^{D}(t)+F_{c}^{K}
$$

where

$$
F_{c}^{D}(t)=D \frac{d x(t)}{d t}
$$

$D$ is the damper coefficient, and

$$
F_{c}^{K}(t)= \begin{cases}K(-x) & \text { if } x<0 \\ 0 & \text { if } 0 \leq x \leq L_{s} \\ -K\left(x-L_{s}\right) & \text { if } x>L_{s}\end{cases}
$$

In Eq. (14), $K$ and $L_{s}$ represent the (large) spring constant associated with the flexible seats and the maximum movement allowed to the moving mass, respectively (Daigle $\&$ Goeble, 2010). The force $F_{p r s}$ in Figure 5 is the pressure force, which is approximated by (Taghizadeh et al., 2009):

$$
F_{\text {prs }}=\left(A_{1}-A_{2}\right) P_{\text {in }}+\left(A_{3}-A_{2}\right) P_{\text {out }}
$$

where $P_{\text {in }}$ and $P_{\text {out }}$ are the pressures at the inlet and outlet ports of the valve and $A_{1}$, $A_{2}, A_{3}$ and $A_{4}$ are different areas on the spool lands which are affected by $P_{\text {in }}$ and $P_{\text {out }}$ in different directions. In the valve studied, the areas $A_{3}$ and $A_{4}$ are approximatively equal (Taghizadeh et al., 2009). Then, $F_{p r s} \cong\left(A_{1}-A_{2}\right) P_{i n}$. Finally, the equilibrium equation for the mass $m$ is given by

$$
m \frac{d^{2} x(t)}{d t^{2}}+F_{c}(t)+D \frac{d x(t)}{d t}+k_{2}\left(x(t)-x_{0}^{2}\right)-k_{1}\left(x(t)-x_{0}^{1}\right)=F_{p r s}-F_{m}
$$




$$
x(0)=x_{0}, \frac{d x(0)}{d x}=x_{0}^{\prime} L_{0} \leq x \leq L_{s}, L_{o} \geq 0
$$

In Eq. (17), $L_{0}$ represents the displacement of the lower position of the moving mass with respect to its nominal position, i.e., $L_{o}=0$. The displacement $x$ refers to the same origin as for $L_{o}$.

\subsubsection{Degradation Model (Step D1)}

In our modelling methodology, the model of the nominal micro-valve behavior is extended to encode the model of the effects of the friction due to the accumulation of debris at the seats of the micro-valve, which has been recognized by our industrial partner as the leading cause of performance reduction in the train braking system. As the debris builds up, it impedes the valve spool movements and prevents it from fully opening (Daigle \& Goebel, 2010). This results in a change in the boundary conditions of the spool valve motion: the larger the degradation level, the larger the value of $L_{0}$. We characterize the friction damage by a change in the friction coefficient $r(t)$, and model the damage progression as (Daigle \& Goebel, 2010):

$$
\frac{d r(t)}{d t}=\omega_{r}\left|F_{f}(t) \frac{d x(t)}{d t}\right|, r(0)=r_{0}
$$

where $\omega_{r}$ is the wear coefficient, whereas $F_{f}(t)$ is the friction force (Daigle \& Goebel, 2010). This is defined as:

$$
F_{f}(t)=r(t) \frac{d x(t)}{d t}
$$

Notice that the value of $\omega_{r}$ can be estimated from real data, once these become available. Finally, Eq. (16a) is modified to encode the damages due to friction:

$$
m \frac{d^{2} x(t)}{d t^{2}}+F_{c}(t)+D \frac{d x(t)}{d t}+k_{2}\left(x(t)-x_{0}^{2}\right)-k_{1}\left(x(t)-x_{0}^{1}\right)=F_{p r s}-F_{m}(t)-F_{f}(t)
$$

From the discussion above, it follows that

$$
L_{0}=L_{0}(r(t))
$$

where $L_{o}$ is a monotone increasing function of the kinetic friction coefficient, that is, the mass can no longer reach the position $x$ where $L_{0}=0$ due to the debris. The maximum elongation allowed to the spool valve

$$
L_{s-o}=L_{s-o}(r(t))=L_{s}-L_{o}
$$

is a decreasing function of $r(t)$. 


\section{DEVELOPMENT OF THE DIAGNOSTIC CLASSIFIER (Steps S1+D1+D2+D3)}

In this Section, a classification algorithm is developed to assess the degradation level of the solenoid valve based on the solenoid current signals obtained from the simulator of the FPM described in Section 3. The FPM parameters have been set according to our partner knowledge and are reported in Tables 1, 2 and 3 below.

\begin{tabular}{|c|c|c|c|c|}
\hline $\boldsymbol{\mu}_{\mathbf{0}}$ & $\boldsymbol{d}$ & $\boldsymbol{l}_{\mathbf{0}}$ & $\boldsymbol{N}$ & $\boldsymbol{R}_{\text {sol }}$ \\
\hline $4 \pi \cdot 10^{-7} \mathrm{H} / \mathrm{m}$ & $7 \cdot 10^{-4} \mathrm{~m}$ & $6.5 \cdot 10^{-4} \mathrm{~m}$ & $3210 \mathrm{ts}$ & $236 \Omega$ \\
\hline
\end{tabular}

Table 1. Values of parameters $\mu_{0}, d, l_{0}, N$ and $R_{\text {sol }}$

\begin{tabular}{|c|c|c|c|c|}
\hline $\boldsymbol{K}_{\mathbf{1}}$ & $\boldsymbol{K}_{\mathbf{2}}$ & $\boldsymbol{x}_{\mathbf{0}}^{\mathbf{1}}$ & $\boldsymbol{x}_{\mathbf{0}}^{\mathbf{2}}$ & $\boldsymbol{m}$ \\
\hline $367.875 \mathrm{~N} / \mathrm{m}$ & $588.6 \mathrm{~N} / \mathrm{m}$ & $7.13 \cdot 10^{-3} \mathrm{~m}$ & $0.967 \cdot 10^{-3} \mathrm{~m}$ & $5.4957 \cdot 10^{-3} \mathrm{Kg}$ \\
\hline
\end{tabular}

Table 2. Values of parameters $K_{1}, K_{2}, x_{0}^{1}, x_{0}^{2}$ and $m$

\begin{tabular}{|c|c|c|c|c|c|c|c|c|}
\hline $\boldsymbol{D}$ & $\boldsymbol{K}$ & $\boldsymbol{L}_{\boldsymbol{s}}$ & $\boldsymbol{F}_{\boldsymbol{p r s}}$ & $\boldsymbol{i}_{\mathbf{0}}$ & $\boldsymbol{x}_{\mathbf{0}}$ & $\boldsymbol{x}_{\mathbf{0}}^{\prime}$ & $\boldsymbol{L}_{\mathbf{0}}$ & $\boldsymbol{\omega}_{\boldsymbol{r}}$ \\
\hline $5 \mathrm{~N} \cdot \mathrm{s} / \mathrm{m}$ & $\begin{array}{l}10^{8} \mathrm{~N} \\
/ \mathrm{m}\end{array}$ & $0.3 \cdot 10^{-3} \mathrm{~m}$ & $0.7442 \mathrm{~N}$ & $0 \mathrm{~A}$ & $0.3 \cdot 10^{-3} \mathrm{~m}$ & $0 \mathrm{~A} / \mathrm{s}$ & 0 & $10^{-10}$ \\
$\mathrm{~m}$
\end{tabular}

Table 3. Values of parameters, $K, L_{s} F_{p r s}, i_{0}, x_{0}, x_{0}^{\prime}$ and $\omega_{r}$

\subsection{Model simulation (Steps S1+D1)}

For the solenoid valve under investigation, we have considered 4 degradation levels: 'normal', 'low damage', 'medium damage' and 'failure', which correspond to an increase in the value of the lower position of the moving mass $L_{0}$ and, consequently, to a decrease in the value of the highest position $L_{s}$, as reported in Table 4 .

\begin{tabular}{|l|c|c|}
\hline \multicolumn{1}{|c|}{ Degradation level } & $\boldsymbol{L}_{\mathbf{0}}(\boldsymbol{m m})$ & $\begin{array}{c}\text { Percentage reduction in } \\
\boldsymbol{L}_{\boldsymbol{o}-\boldsymbol{s}}(\boldsymbol{p} \boldsymbol{r})\end{array}$ \\
\hline Normal & $0 \leq L_{0}<0.02$ & $0 \% \leq p r<6.67 \%$ \\
\hline Low Damage & $0.02 \leq L_{0}<0.04$ & $6.67 \% \leq p r<13.34 \%$ \\
\hline Medium Damage & $0.04 \leq L_{0}<0.06$ & $13.37 \% \leq p r<20 \%$ \\
\hline Failure & $L_{0} \geq 0.06$ & $p r \geq 20 \%$ \\
\hline
\end{tabular}

Table 4. Threshold values of $L_{0}$ and $p r$ characterizing the 4 degradation levels

To simulate different levels of degradation, we need to find the values of the kinetic friction coefficient $r(t)$ associated to the threshold values in Table 5, which refer to the valve spool displacement. To establish the relationships between the displacement and the friction coefficient, we have simulated 2000 solenoid current signals, corresponding to the values $\{1, \ldots, 2000\}$ of the initial condition $r_{0}$ in Eqs. (18) with $\omega_{r}=0.00001$ and the input voltage coming from real measurements. For each simulated current profile, we have got the corresponding value of $L_{0}$ : this gives the curve $\left(r_{0}, L_{0}\left(r_{0}\right)\right)$ shown in Figure 6, which maps the valve spool position onto the degradation model. Different valve spool positions correspond to different flow rate values, and thus different breaking performances. From this, we can derive the upper bounds $r_{n}, r_{l}$ and $r_{m}$ for defining the 'normal', 'low damage' and 'medium damage' degradation levels, 
respectively (Table 5), which correspond to decreasing flow rate levels and, thus, decreasing valve performance.

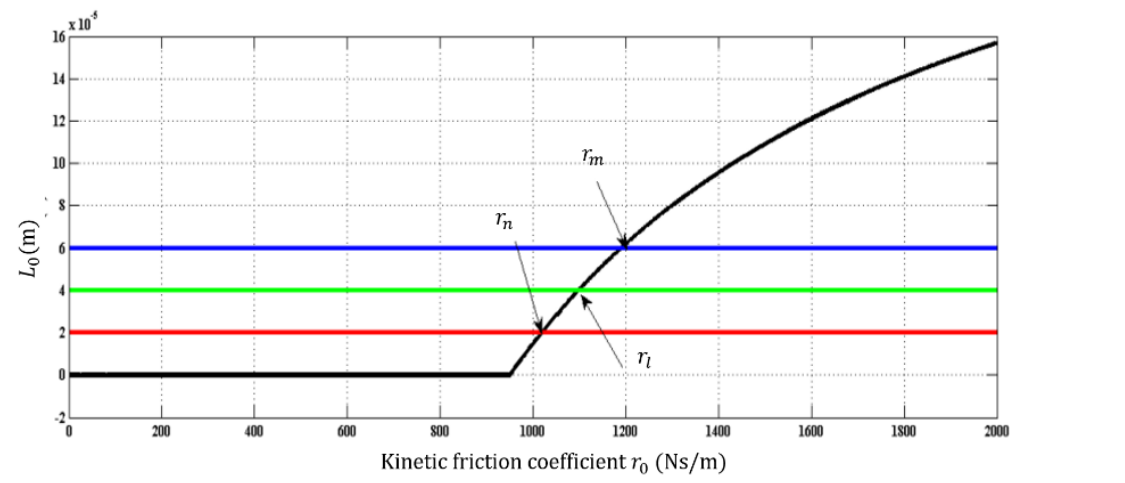

Fig. 6. Kinetic friction coefficient $\boldsymbol{r}_{\mathbf{0}}$ vs $\boldsymbol{L}_{\mathbf{0}}$

\begin{tabular}{|c|c|c|c|}
\hline $\boldsymbol{I}_{\mathbf{0}}$ (normal) & $\boldsymbol{I}_{\mathbf{1}}$ (low damage) & $\boldsymbol{I}_{\mathbf{2}}$ (medium damage) & $\boldsymbol{I}_{\mathbf{3}}$ (failure) \\
\hline$\left(0, r_{n}\right)=(0,1018)$ & {$\left[r_{n}, r_{l}\right)=[1019,1097)$} & {$\left[r_{l}, r_{m}\right)=[1098,1189)$} & {$\left[r_{m}, 2000\right)=[1189,2000]$} \\
\hline
\end{tabular}

Table 5. Kinetic friction value intervals corresponding to the four degradation levels

\subsection{Data history matrix generation (Steps S1+D1)}

We have randomly generated 200 values of $r_{0}$ within each interval (see Table 5), and we have simulated the FPM model. By so doing, we have obtained a total of 800 solenoid current signals, labelled as $g_{1_{i}}, g_{2_{i}}, g_{3_{i}}$ and $g_{4_{i}} i=1, \ldots, 200$, depending on the degradation state in which they are generated: normal, low damage, medium damage and failure, respectively. Finally, we have also added a white noise to all 800 generated current signals, to take into account that real data are expected to be noisy (e.g., owing to current sensor noise).

\subsection{Feature extraction (Step D2)}

In the case study proposed in this work, a set of $n=177$ features have been extracted (see Appendix B) from each current signal, which include statistical indicators (features 1 to 9), Wavelet Transforms (WPT) using different basis (Biorthogonal 2.4 (features 10 to 51), Reverse Biorthogonal 2.4 (features 52 to 93), Symlet5 basis (features 94 to 135) and Haar basis (features 136-177)) (Baraldi et al, 2016).

By so doing, we have obtained a dataset $X$ consisting of 800 patterns in the 177dimensional space. Since the range of signal values of the entries of the matrix $X$ can widely vary, these have been normalized: the values in every column of $X$ have been 
re-scaled so that they are (standard) normally distributed with mean equal to 0 and standard deviation equal to 1 .

Then, all the available 800 labelled data of $X$ are partitioned into

- A set used for the feature selection task, which is made up of $50 \%$ of the total number of patterns, obtained by randomly sampling 100 patterns among the 200 patterns available at each degradation level.

- A validation set, which contains the remaining patterns. These will be used for validating the performance of the diagnostic model after the optimal features subset selection.

\subsection{Feature Selection (Step D2)}

The problem of selecting from the set of features extracted an optimal subset of features relevant for classification can be framed as a Multi-objective (MO) optimization problem. Namely, the wrapper approach builds a number of candidate features set $\boldsymbol{z} \in\{0,1\}^{n}$, where $z(k)=1$ denotes that feature $k$ is selected, whereas $z(k)=0$ that it is not selected. The performance of each feature set $\boldsymbol{z}$ is evaluated with respect to a multi-objective fitness function $\boldsymbol{F}$, which is defined as:

$$
\boldsymbol{F}=\left[-F_{1}(\mathbf{z}), F_{2}(\mathbf{z})\right] \boldsymbol{z} \in\{0,1\}^{n}
$$

where $F_{2}(\cdot)$ counts the number of features (to be minimized) forming the subsets:

$$
F_{2}(\mathbf{z})=\sum_{k=1}^{n} z(k)
$$

whereas $F_{1}(\cdot)$ represents the classification accuracy of the diagnostic classifier (in Eq. (23) the sign is changed, as we use a DE algorithm for minimization). To calculate this value, the total number of pre-labelled available patterns is randomly subdivided into training set and test set, consisting of $75 \%$ and $25 \%$ of the data, respectively. The random subdivision of the available patterns in training and test sets is repeated 20 times (i.e., 20 cross-validations): the mean recognition rate, i.e., the average fraction of correct classifications over the 20 cross-validation tests, is then calculated and represents the fitness value $F_{1}$ of candidate solution $\boldsymbol{z}$. Finally, the classification algorithm used in this work is the Fuzzy K-Nearest Neighbor (FKNN) algorithm.

The performance of the MO optimization can be quantified in terms of the diversity of the solutions and the convergence to the Pareto optimal front (Baraldi et al, 2016). Since in a MO optimization problem, it is typically not possible to simultaneously improve the values of two or more objective functions without causing the deterioration of some other objectives, diversity is a fundamental requirement in a MO evolutionary optimization. In practice, diversity in the population allows improving the coverage of the search space and exploring different evolutionary paths. An indicator of the diversity of a Pareto optimal set is the hyper-volume over the dominated set, which has been defined as the Lebesgue-measure of the hyper-volume with respect to a lower reference bound (normally, the ideal best values of each objective function) (Baraldi et al., 2016): the smaller is the value of such indicator, the better is the performance in terms of objective function evaluations and the wider is the exploration of the search 
space. In our case, we set $(-1,0)$ as the smaller reference point (i.e., the feature set characterized by the best possible performances), which corresponds to the situation where the patterns are all classified correctly $\left(-F_{1}(\mathbf{z})=-1\right)$ and $F_{2}(\mathbf{z})=0$ features are used.

Figure 7 shows that the optimal Pareto set hyper-volume significantly decreases until (approximatively) generation 200; then, it remains constant. This indicates that the Pareto set becomes stable and no improvement is expected in the solution optimal set by further increasing the number of generations.

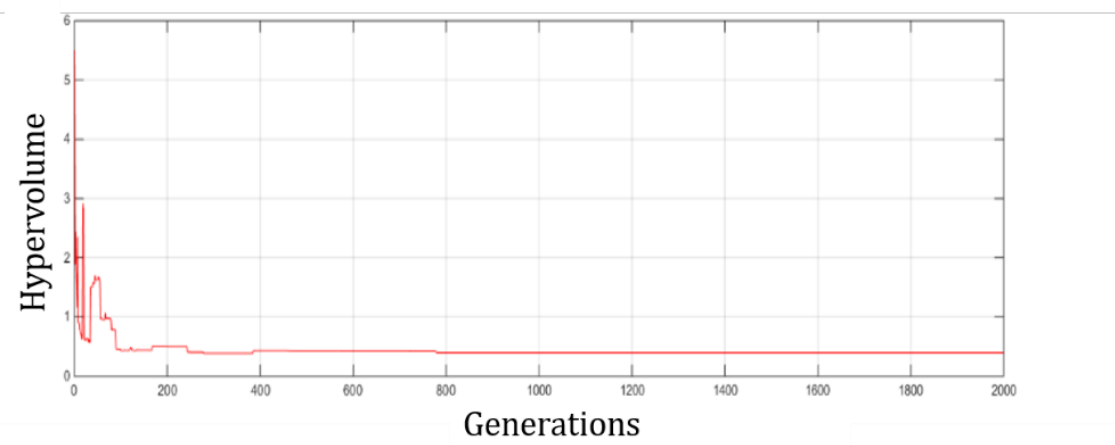

Fig. 7. Hyper-volume values iteration by iteration

In Table 6, the candidate solutions in the Pareto optimal set are reported.

\begin{tabular}{|c|c|c|}
\hline Solution & $\begin{array}{c}\text { Classification accuracy } \\
\left(\boldsymbol{F}_{\mathbf{2}}\right)\end{array}$ & $\begin{array}{c}\text { Number of features } \\
\left(\boldsymbol{F}_{\mathbf{1}}\right)\end{array}$ \\
\hline $\mathbf{z}_{1}^{B}$ & 0.9469 & 1 \\
\hline $\mathbf{z}_{2}^{B}$ & 0.9673 & 2 \\
\hline $\mathbf{z}_{3}^{B}$ & 0.9781 & 3 \\
\hline $\mathbf{z}_{4}^{B}$ & 0.9816 & 4 \\
\hline $\mathbf{z}_{5}^{B}$ & 0.9837 & 5 \\
\hline $\mathbf{z}_{6}^{B}$ & 0.9847 & 6 \\
\hline $\mathbf{z}_{7}^{B}$ & 0.9852 & 7 \\
\hline $\mathbf{z}_{8}^{B}$ & 0.9857 & 10 \\
\hline
\end{tabular}

Table 6. Candidate solution in the Pareto optimal front

We have chosen the solution with best classification accuracy, i.e., solution $\boldsymbol{z}_{8}^{B}$, disregarding the number of features. This choice is motivated by the fact that an increment in the number of features does not significantly increase the computational burden. We have developed the Fuzzy K-Nearest Neighbours (FKNN) to classify the solenoid valve degradation states. After the feature selection task, the solenoid valve classification system has been developed. The overall accuracy of the classification 
model obtained in a 20 -folds cross validation on the data of the validation set (i.e., containing data not previously used during the feature selection) is $0.9671 \pm 0.0133$ which is satisfactory.

\section{Conclusions}

In this work, we have proposed a diagnostic tool based on FPM simulator to cope realistic case in which field data of the system in normal and abnormal condition are not currently available. To show the procedural steps of the approach, an application has been presented with reference to fault diagnostic of a solenoid valve mounted on the braking system of trains.

Future works will be focussed on the further application of the proposed methodology to the solenoid valve pilot case study, to make use of field data as they become available, for:

1. Updating the fault history matrix, the parameters of the FPM and the diagnostic model (steps D4-D5-D6, respectively).

2. Extend the proposed methodology to fault prognostics and developing a prognostic model for solenoid valve.

3. Making uncertainty analysis (steps D7).

\section{References}

Aster, R.C., Borchers, B., Thurber, C.H. (2013). Parameter Estimation and Inverse Problems. Parameter Estimation and Inverse Problems, Academic Press.

Aven, T., Baraldi, P., Flage, R., Zio, E. (2014). Uncertainty in Risk Assessment: The Representation and Treatment of Uncertainties by Probabilistic and Non-Probabilistic Methods, 1-186, 2014.

Baraldi, P., Canesi, R., Zio, E., Seraoui, R., Chevalier, R. (2011). Genetic algorithmbased wrapper approach for grouping condition monitoring signals of nuclear power plant components. Integrated Computer-Aided Engineering, 18 (3): 221-234.

Baraldi, P., Cannarile, F., Di Maio, F., Zio, E. (2016). Hierarchical k-nearest neighbours classification and binary differential evolution for fault diagnostics of automotive bearings operating under variable conditions. Engineering Applications of Artificial Intelligence, 56, pp. 1-13.

Daigle, M., Goebel, K. (2010). Improving computational efficiency of prediction in model-based prognostics using the unscented transform. Proceedings of the Annual Conference of the Prognostics and Health Management Society.

Hastie T. J. Tibshirani R. J. \& Friedman J. H. (2009). The Elements of Statistical Learning. New York: Springer, 2nd ed. 
Kantas, N., Doucet, A., Singh, S.S., Maciejowski, J., Chopin, N (2015). On particle methods for parameter estimation in state-space models. Statistical Science, 30 (3), pp. 328-351.

Kühl, P., Diehl, M., Kraus, T., Schlöder, J.P., Bock, H.G. (2011). A real-time algorithm for moving horizon state and parameter estimation. Computers and Chemical Engineering, 35 (1), pp. 71-83.

Pantelides, C.C., Renfro, J.G (2013). The online use of first-principles models in process operations: Review, current status and future needs. Computers and Chemical Engineering, 51, pp. 136-148.

Pecht, M.G. (2008). Prognostics and Health Management of Electronics. Prognostics and Health Management of Electronics, pp. 1-315.

Rizzoni, G., (2007). Principles and Applications of Electrical Engineering, 5/e, McGraw Hill, 2007.

Taghizadeh, M., Ghaffari, A., Najafi, F. (2009). Modeling and identification of a solenoid valve for PWM control applications. Comptes Rendus Mecanique, 337 (3):131-140.

Zio, E. (2012). Prognostics and health management of industrial equipment. Diagnostics and Prognostics of Engineering Systems: Methods and Techniques, 333356. 